\title{
Pacific
}

Journal of

Mathematics

\section{ON ISOTROPIC SUBMANIFOLDS AND EVOLUTION OF QUASICAUSTICS}

\author{
STANISŁAW JANECZKO
}




\title{
ON ISOTROPIC SUBMANIFOLDS AND EVOLUTION OF QUASICAUSTICS
}

\author{
STANISLAW JANECZKO ${ }^{1}$
}

\begin{abstract}
We study classification problems for generic isotropic submanifolds. The classification list of simple and unimodal singularities is obtained and the generic evolutions of quasicaustics in small dimension are classified. Examples encountered in geometric optics are presented.
\end{abstract}

0. Introduction and preliminaries. Let $X$ be a manifold, and $\omega$ be a 2-form on $X$. The pair $(X, \omega)$ is called a symplectic manifold if $\omega$ is closed, i.e. $d \omega=0$ and nondegenerate [AM]. The representative model of a symplectic manifold is a cotangent bundle $T^{*} M$, endowed with the canonical 2-form $\omega_{M}=d \vartheta_{M}$, where the 1-form $\vartheta_{M}$ on $T^{*} M$ (Liouville form) is defined by

$$
\left\langle u, \vartheta_{M}\right\rangle=\left\langle T \pi_{M}(u), \tau_{T^{*} M}(u)\right\rangle, \quad \text { for each } u \in T T^{*} M .
$$

The mapping $T \pi_{M}$ is the tangent mapping of $\pi_{M}: T^{*} M \rightarrow M$ and $\tau_{T^{*} M}: T T^{*} M \rightarrow T^{*} M$ is the tangent bundle projection. If $\left(q_{i}\right)$ are local coordinates introduced in $M$, and $\left(p_{i}, q_{i}\right)$ are corresponding local coordinates in $T^{*} M$ then $\omega_{M}$ has the normal (Darboux) form $\omega_{M}=\sum_{i=1}^{n} d p_{i} \wedge d q_{i}[\mathbf{W e}]$.

We recall that a submanifold $C \subset(X, \omega)$ is coisotropic if, at each $x \in C$, the symplectic polar of $T_{x} C$ defined by

$$
C_{x}^{\perp}=\left\{v \in T_{x} X:\langle v \wedge u, \omega\rangle=0 \text { for every } u \in T_{x} C\right\}
$$

is contained in $T_{x} C$. By $\langle v \wedge u, \omega\rangle$ we denote the evaluation of $\omega$ on the pair of vectors $v, u \in T_{x} X$. If $C_{x}^{\perp}=T_{x} C$ for each $x \in C$ then $C$ is called the Lagrangian submanifold of $X$. In this case $\left.\omega\right|_{C}=0$, and $\operatorname{dim} C=\frac{1}{2} \operatorname{dim} X$. We see that $\operatorname{dim} C_{x}^{\perp}=\operatorname{codim} C$ and $\left\{C_{X}^{\perp}\right\}$ forms the characteristic distribution of $\left.\omega\right|_{C}$. Thus the distribution $D=\bigcup_{x \in C} C_{\bar{x}}^{\perp}$ is involutive. Maximal connected integral manifolds of $D$ are called bicharacteristics. They form the characteristic foliation of $C$ (cf. [AM]). $D$ represents the generalized Hamiltonian system

${ }^{1}$ On leave from Mathematics Institute, Technical University of Warsaw, Pl. Politechniki 1, 00-661 Warsaw, Poland. 
with "Hamiltonian" $C$. Let $Y$ be the set of bicharacteristics of $C$. Let $\rho: C \rightarrow Y$ be canonical projection along bicharacteristics and we define the graph of $\rho$,

$$
\mathscr{R}_{C}=\operatorname{graph} \rho=\{(x, y) \in X \times Y ; y=\rho(x), x \in X\} .
$$

If $Y$ admits a differentiable structure and $\rho$ is a submersion, then there is a unique symplectic structure $\kappa$ on $Y$ such that

$$
\rho^{*} \kappa=\left.\omega\right|_{C} .
$$

Thus we deduce that $\mathscr{R}_{C}$ is a Lagrangian submanifold of $X \times Y$ endowed with the symplectic structure $\Omega=\pi_{2}^{*} \kappa-\pi_{1}^{*} \omega$, where $\pi_{i}: X \times$ $Y \rightarrow X(Y), i=1,2$, are the cartesian projections. In fact $\left.\Omega\right|_{\mathscr{R}_{C}}=$ $\rho^{*} \kappa-\left.\omega\right|_{C}=0$ and $\operatorname{dim} \mathscr{R}_{C}=\frac{1}{2}(\operatorname{dim} X+\operatorname{dim} Y)$. Being the graph of submersion, $\mathscr{R}_{C}$ is called the symplectic reduction relation between $(X, \omega)$ and $(Y, \kappa)$. More generally we define a symplectic relation $\mathscr{R}$ from $(X, \omega)$ to $(Y, \kappa)$ as an immersed Lagrangian submanifold of the symplectic manifold $(X \times Y, \Omega)$. For any subset $\mathscr{F} \subset X$, the set $\mathscr{R}(\mathscr{F})=\{y \in Y$; there exists $x \in \mathscr{F}$ such that $(x, y) \in \mathscr{R}\}$ is called the image of $\mathscr{F}$ with respect to the relation $\mathscr{R}$. If $\mathscr{S} \subset Y$ then the set $\mathscr{R}^{t}(\mathscr{S}) \subset X$ is called the counterimage of $\mathscr{S}$ with respect to $\mathscr{R}$. Here $R^{t}$ is the transposed relation $\mathscr{R}^{t}=\{(y, x) \in Y \times X$; $(x, y) \in \mathscr{R}\} \subset\left(Y \times X, \pi_{1}^{*} \omega-\pi_{2}^{*} \kappa\right)$.

Let $L$ be a Lagrangian submanifold of $\left(T^{*} M, \omega_{M}\right)$ transversal, in a neighborhood of some point $p \in L$, to the fibers of the canonical fibration $\pi_{M}$. Then in a neighborhood of the point $\pi_{M}(p) \in M$ there exists a smooth function $S: M \rightarrow R$ such that $L$ is locally defined as the graph of the section $d S: M \rightarrow T^{*} M . S$ is called the generating function of $L$ (cf. [Hör]). If the transversality condition is not fulfilled then $L$ is represented locally as an image $\mathscr{R}_{C}(N)$ by the symplectic reduction relation $\mathscr{R}_{C}, C \subset T^{*}(M \times \Lambda), C=\{(p, q, \mu, \lambda) ; \mu=0\}$, where $\Lambda \cong R^{k}$ is a Morse parameter space parametrized by $(\lambda)$, and $N$ is a Lagrangian submanifold of $T^{*}(\Lambda \times M)$ transversal to the canonical fibration. Thus $L$ is always locally represented by a family $F$, of functions on a manifold $\Lambda$, parametrized by $M ; F: M \times \Lambda \rightarrow$ $R$ (cf. [Wei]). It is called the Morse family or generating family, and defines $L$ by the following equations:

$$
\begin{aligned}
& L=\left\{(p, q) \in T^{*} M ; \text { there exists } \lambda \in \Lambda,\right. \text { such that } \\
& \qquad p=\partial F / \partial q(q, \lambda) \text { and } 0=\partial F / \partial \lambda(q, \lambda)\},
\end{aligned}
$$

near $p \in T^{*} M$. The mapping $d_{\lambda} F: M \times \Lambda \rightarrow \Lambda$, is assumed to have maximal rank at $\left(\pi_{M}(p), 0\right) \in M \times \Lambda$. The set of critical values of the 
Lagrange projection $\left.\pi_{M}\right|_{L}: L \rightarrow M$ is called the caustic of $L$. The corresponding theory of generating families for coisotropic varieties is presented in [Ja3]. In what follows we construct the generating family approach to another important class of objects of symplectic geometry called isotropic submanifolds (cf. [Wei], p. 4).

Let $I$ be an isotropic submanifold of $\left(T^{*} M, \omega_{M}\right)$; i.e. if $i: I \rightarrow$ $T^{*} M$ is an immersion of $I$ then $i^{*} \omega_{M}=0$. We assume $\operatorname{dim} I<$ $\operatorname{dim} M$, then the quasicaustic of $I$ is defined as an image $\pi_{M}(I)$.

Isotropic submanifolds and their quasicaustics arise naturally in a number of contexts including, for example, optical diffraction on apertures [Kel], [Ja2], geometry of bicharacteristics of Hamilton-Jacobi equations $[\mathbf{A M}]$ and symmetric phase transitions [JR]. In this paper we introduce the notion of generating families for isotropic submanifolds. Then we find that the natural group of equivalences, in the space of generating families, preserves the boundaries and corners in Morse parameters (cf. [Sie]). In contrast to the equivalences of the coisotropic submanifolds, which preserve the fibre structure in unfolding parameters (cf. [Was], [Ja3]), our group comes from the straightforward generalization of the standard singularity theory (cf. [Wa1]).

In $\S 1$, we introduce the notion of $I$-Morse family generating an isotropic submanifold $I$ and show geometric examples where isotropic submanifolds and their generating families appear naturally.

In $\S 2$, we describe the general singularity theory machinery that can be used to classify isotropic submanifolds and their quasicaustics. Then we classify the simple and unimodal $I$-Morse families generating the isotropic submanifolds with $\operatorname{dim} I=\operatorname{dim} M-1$, which involve maximally three Morse parameters.

In $\S 3$, we apply the methods of singularity theory of functions on varieties [Bru], giving the complete classification of generic evolutions of quasicaustics that can occur if $\operatorname{dim} M<4$.

Acknowledgments. I would like to thank Ted Courant, Richard Montgomery and Tudor Ratiu for helpful discussions.

1. Generating families for isotropic varieties. Let $I$ be a connected submanifold of a symplectic manifold $(X, \omega)$. If for each $x \in I$,

$$
I_{x}^{\perp}=\left\{v \in T_{x} X:\langle v \wedge u, \omega\rangle=0 \text { for every } u \in T_{x} I\right\} \supset T_{x} I,
$$

we call $I$ an isotropic submanifold of $X$. If $i: I \rightarrow X$ is an immersion of $I$ then $I$ is called isotropic if $i^{*} \omega=0$ (cf. [AM]). It follows that $\operatorname{dim} I \leq \frac{1}{2} \operatorname{dim} X$, and lagrangian submanifold is the case $I_{x}^{\perp}=T_{x} I$. 
Let $I$ be an isotropic submanifold of $\left(T^{*} M, \omega_{M}\right), \operatorname{dim} I<\operatorname{dim} M$. Following the idea that all symplectic objects should be, at least locally, generated by generating families we seek an adequate notion corresponding to isotropic varieties.

Definition 1.1. The smooth function (germ) $G: M \times R^{L} \times R^{K} \rightarrow R$ is called an $I$-Morse family if the smooth map

$$
M \times R^{K} \ni(q, \lambda) \rightarrow\left(\frac{\partial G}{\partial \beta}(q, 0, \lambda), \frac{\partial G}{\partial \lambda}(q, 0, \lambda)\right) \in R^{L} \times R^{K}
$$

is nonsingular on the stationary set

$$
\Sigma_{G}^{I}=\left\{(q, \lambda): \frac{\partial G}{\partial \beta}(q, 0, \lambda)=0, \frac{\partial G}{\partial \lambda}(q, 0, \lambda)=0\right\} .
$$

Let $G$ be an $I$-Morse family; then it is easy to check that the set

(2) $I=\left\{(p, q) \in T^{*} M\right.$ : there exists $\lambda$ such that

$$
\left.p=\frac{\partial G}{\partial q}(q, 0, \lambda), \frac{\partial G}{\partial \beta}(q, 0, \lambda)=0=\frac{\partial G}{\partial \lambda}(q, 0, \lambda)\right\}
$$

is a smooth immersed isotropic submanifold of $T^{*} M$. If we drop the conditions of Definition 1.1, then the corresponding set defined by (2) may be a singular isotropic variety. In this case we call the function $G$ a generating family for $I$.

Now we have an analog of the theorem on local generating families for lagrangian submanifolds (cf. [Wei], Ch. 6). We assume $M \equiv R^{N}$.

Proposition 1.2. To each germ of an immersed isotropic submanifold $(I, 0) \subset T^{*} M$, there exists a germ of an I-Morse family

$$
G:\left(M \times R^{L} \times R^{K},(0,0,0)\right) \rightarrow R
$$

such that $(I, 0)$ is defined by (2).

Proof. To each germ $(I, 0)$ corresponds a coisotropic submanifold $(C, 0) \subset T^{*} M$ with $I$ to be its bicharacteristic passing through 0 . Let $\rho: C \rightarrow T^{*} R^{L}$ denote the canonical projection along bicharacteristics of $C$. Then there exists a Morse family $G: M \times R^{L} \times R^{K} \rightarrow R$ for graph $\rho \subset\left(T^{*} M \times T^{*} R^{L} ; \pi_{2}^{*} \omega_{R^{L}} \ominus \pi_{1}^{*} \omega_{M}\right)$, such that $I$ is the image of the mapping

$$
\Sigma_{G}^{I} \ni(q, \lambda) \rightarrow\left(\frac{\partial G}{\partial q}(q, 0, \lambda), q\right) \in T^{*} M
$$


Let $R^{N} \times R^{S} \times R^{K} \ni(q, \mu, \lambda) \rightarrow G(q, \mu, \lambda)$ be a Morse family generating the symplectic relation $R$ in $\left(T^{*} R^{N} \times T^{*} R^{S}, \omega_{R^{s}} \ominus \omega_{R^{N}}\right)$ (cf. [Ja2]). Then we immediately have the following result.

Proposition 1.3. Assume that the mapping

$$
R^{N} \times R^{S} \times R^{K} \ni(q, \mu, \lambda) \rightarrow\left(\frac{\partial G}{\partial \mu}(q, \mu, \lambda), \frac{\partial G}{\partial \lambda}(q, \mu, \lambda)\right)
$$

has a maximal rank on the stationary set $\Sigma_{G}=\left\{(q, \mu, \lambda): \frac{\partial G}{\partial \lambda}(q, \mu, \lambda)\right.$ $=0\}$. Then the family

$$
R^{N} \times R^{W} \times R^{S-W} \times R^{K} \ni(q, \beta, \bar{\mu}, \lambda) \rightarrow G(q, \beta, \bar{\mu}, \lambda)
$$

is an I-Morse family generating the isotropic submanifold defined as an image of $\left\{(\nu, \mu) \in T^{*} R^{S}: \nu=0, \mu_{1}=0, \ldots, \mu_{W}=0\right\}$ by the transposed symplectic relation $R^{t}$.

EXAMPLe 1.4. Systems of rays by diffraction on apertures. Let $X \equiv$ $R^{n}$ be the configuration space of geometric optics. Let $S$ be a hypersurface with boundary $\partial S$ in $X$, representing the aperture of an optical system (cf. [Ja2]). Let $\Psi$ be an initial wavefront hypersurface in $X$ with coordinates $\left(\mu_{1}, \ldots, \mu_{n-1}\right)$. Then all rays passing through the boundary of an aperture $\partial S$ form an isotropic subvariety $I^{n-1}$ of $\left(T^{*} X, \omega_{X}\right)$ (this is also an isotropic subvariety of the symplectic space of all rays [Ja2]). $I^{n-1}$ is generated by the distance function, say $G(x, \beta, \lambda, \mu)$ from the point $\mu=\left(\mu_{1}, \ldots, \mu_{n-1}\right)$ of the wavefront to the point $(0, \beta, \lambda)$ of the complement of the aperture $\bar{S}=\left\{\left(0, \beta, \lambda_{1}, \ldots, \lambda_{n-2}\right) \in X ; \beta \geq 0\right\},\left(\lambda=\left(\lambda_{1}, \ldots, \lambda_{n-2}\right)\right)$, plus the distance from the point $(0, \beta, \lambda) \in \bar{S}$ to the final fixed point $x \in X$. In this case $L=1, K=2 n-3$ and generically $G$ is an $I$-Morse family.

There are isotropic varieties which play an important role in geometry and physics (see [Kel], [Hor], [AG], [Ja2]) and they are no longer smooth. Nevertheless they can still be represented in the form (2) by the $I$-generating families.

EXAMPLE 1.5. Isotropic varieties of polynomials. Let us consider the space of polynomials.

$$
\begin{aligned}
\left\{\frac{x^{2 k+2}}{(2 k+2) !}\right. & +\bar{q}_{1} \frac{x^{2 k+1}}{(2 k+1) !}+\cdots+\bar{q}_{k+1} \frac{x^{k+1}}{(k+1) !}-\bar{p}_{k+1} \frac{x^{k}}{k !} \\
& \left.+\cdots+(-1)^{l} \bar{p}_{k+2-l} \frac{x^{k+1-l}}{(k+1-l) !}+\cdots+(-1)^{k+1} \bar{p}_{1}\right\},
\end{aligned}
$$


endowed with the symplectic structure $\omega=\sum_{i=1}^{k+1} d \bar{p}_{i} \wedge d \bar{q}_{i}$ derived from the unique $\mathrm{SL}_{2}(R)$-invariant symplectic structure of the space of binary forms of $2 k+3$-degree by symplectic reduction (see [Zak], [AR1]). The space of characteristics of the Hamiltonian system with Hamiltonian $H(p, q)=\bar{p}_{1}+\bar{q}_{1} \bar{p}_{2}+\cdots+\frac{1}{2} \bar{q}_{k+1}^{2}$, corresponding to translations of $x$, is identified with the space of polynomials

$$
\begin{aligned}
T^{*} Q=\left\{\frac{x^{2 k+1}}{(2 k+1) !}\right. & +q_{1} \frac{x^{2 k-1}}{(2 k-1) !} \\
& \left.+\cdots+q_{k} \frac{x^{k}}{k !}-p_{k} \frac{x^{k-1}}{(k-1) !}+\cdots+(-1)^{k} p_{1}\right\},
\end{aligned}
$$

endowed with the reduced symplectic form $\sum_{i=1}^{k} d p_{i} \wedge d q_{i}$. By the obvious identification

$$
\begin{aligned}
\frac{(x-t)^{2 k+2}}{(2 k+2) !} & +\bar{q}_{1} \frac{(x-t)^{2 k+1}}{(2 k+1) !}+\cdots+\bar{q}_{k+1} \frac{(x-t)^{k+1}}{(k+1) !}-\bar{p}_{k+1} \frac{(x-t)^{k}}{k !} \\
& +\cdots+(-1)^{k+1} \overline{p_{1}} \\
= & \frac{x^{2 k+2}}{(2 k+2) !}+q_{1} \frac{x^{2 k}}{(2 k) !}+\cdots+q_{k} \frac{x^{k+1}}{(k+1) !}-p_{k} \frac{x^{k}}{k !} \\
& +\cdots+(-1)^{k}\left(\sum_{i=1}^{k-1} q_{i} p_{i+1}+\frac{1}{2} q_{k}^{2}\right)
\end{aligned}
$$

we obtain the $I$-generating families for the isotropic spaces of polynomials $I_{r}$ in $T^{*} Q$ having root of multiplicity $\geq k+1+r$. Recall that $I_{0}$ is a lagrangian variety in $T^{*} Q$ called an open swallowtail (cf. [Ar1]).

$$
\begin{aligned}
I_{r}: \quad G_{r}: Q \times R^{r} \times R^{r+1} \rightarrow R, & \left.-\frac{1}{(k-r+\xi) !} \lambda_{r+1}^{k-r+\xi}\right) \\
G_{r}(q, \beta, \lambda)= & \sum_{\xi=1}^{r} \lambda_{\xi}\left(\beta_{\xi}-\sum_{l=1}^{k-r+\xi-1} q_{l} \lambda_{r+1}^{k-r+\xi-l-1} \frac{1}{(k-r+\xi-l-1) !}\right. \\
& -\frac{1}{2} \int_{0}^{\lambda_{r+1}}\left(\frac{1}{(k+1) !} t^{k+1}+\sum_{\rho=1}^{k} q_{\rho} t^{k-\rho} \frac{1}{(k-\rho) !}\right)^{2} d t
\end{aligned}
$$

In the case $k=2, r=1$, i.e. the variety of polynomials of fifth degree having root of multiplicity $\geq 4$, the stationary set is described 
by the three equations

$$
\Sigma_{G_{1}}^{I}=\left\{(q, \lambda) \in Q \times R^{2} ; \lambda_{1}=0,2 q_{1}+\lambda_{2}^{2}=0, \lambda_{2}^{3}+q_{1} \lambda_{2}+q_{2}=0\right\} .
$$

EXAMPLE 1.6. Generalized open Whitney umbrellas. Consider the space of pairs of binary forms of degree $2 n+1,(f, g) \in M^{2 n+2} \times$ $M^{2 n+2}$,

$$
f=\sum_{k=0}^{2 n+1}\left(\begin{array}{c}
2 n+1 \\
k
\end{array}\right) a_{k} x^{k} y^{2 n+1-k}, \quad g=\sum_{i=0}^{2 n+1}\left(\begin{array}{c}
2 n+1 \\
i
\end{array}\right) b_{i} x^{i} y^{2 n+1-i}
$$

By the lineo-linear invariant $\{f, g\}$ in the space of binary forms (cf. [KR]), we define the bilinear form

$$
\omega\left((f, g),\left(f^{\prime}, g^{\prime}\right)\right)=\left(\left\{f, g^{\prime}\right\}-\left\{f^{\prime}, g\right\}\right)(2 n+1) !
$$

which endows the space $M^{2 n+2} \times M^{2 n+2}$ with the $\mathrm{SL}_{2}(R)$ invariant symplectic structure. We recall

$$
\{f, g\}=\sum_{k=0}^{2 n+1}(-1)^{2 n+1-k}\left(\begin{array}{c}
2 n+1 \\
k
\end{array}\right) a_{k} b_{2 n+1-k} .
$$

In the corresponding Darboux coordinate we write

$$
\begin{aligned}
& f=q_{0} \frac{x^{2 n+1}}{(2 n+1) !}+q_{1} \frac{x^{2 n}}{(2 n) !}+\cdots+q_{2 n+1} y^{2 n+1}, \\
& g=p_{2 n+1}(-1)^{2 n+2} \frac{x^{2 n+1}}{(2 n+1) !}+\cdots+p_{1}(-1)^{2} x y^{2 n}+p_{0}(-1)^{1} y^{2 n+1} .
\end{aligned}
$$

By the symplectic reduction on hypersurface $\left\{q_{0}=1\right\}$ we obtain the reduced symplectic space identified with the pairs of polynomials $(\bar{f}, \bar{g}) \in Q^{2 n+1} \times Q^{2 n}$ (we put $y=1$ and consider the derivatives of $g$-polynomial)

$$
\begin{aligned}
& \bar{f}=\frac{x^{2 n+1}}{(2 n+1) !}+q_{1} \frac{x^{2 n}}{(2 n) !}+\cdots+q_{2 n+1}, \\
& \bar{g}=p_{2 n+1}(-1)^{2 n} \frac{x^{2 n}}{(2 n) !}+\cdots+p_{1}(-1)^{0},
\end{aligned}
$$

endowed with the Darboux reduced symplectic structure

$$
\bar{\omega}=\sum_{i=1}^{2 n=1} d p_{i} \wedge d q_{i}
$$

The corresponding Hamiltonian of translations in $\left(Q^{2 n+1} \times Q^{2 n}, \bar{\omega}\right)$ is

$$
H=p_{1}+q_{1} p_{2}+\cdots+q_{2 n} p_{2 n+1} \text {. }
$$


Its space of hamiltonian curves is identified with the space of pairs of polynomials, $(F, G) \in X$

$$
\begin{aligned}
& F=\frac{x^{2 n+1}}{(2 n+1) !}+\bar{q}_{1} \frac{x^{2 n-1}}{(2 n-1) !}+\cdots+\bar{q}_{2 n}, \\
& G=\bar{p}_{2 n}(-1)^{2 n-1} \frac{x^{2 n-1}}{(2 n-1) !}+\cdots+\bar{p}_{1}(-1)
\end{aligned}
$$

with $\omega=\sum_{i=1}^{2 n} d \bar{p}_{i} \wedge d \bar{q}_{i}$. It defines the symplectic mapping along characteristics of $H$, say $\pi_{H}: C=\{H=0\} \rightarrow X$. The coisotropic submanifold $C$ is generated by the following generating family (cf. [Ja3]) $F: Q^{2 n+1} \times R^{2 n} \times R^{2 n} \rightarrow R$,

$$
F(q, \alpha, \lambda)=\sum_{i=2}^{2 n+1} \lambda_{i}\left(q_{i}-\sum_{l=2}^{i} \alpha_{l-1} \frac{1}{(i-l) !} q_{1}^{i-l}-\frac{1}{i !} q_{1}^{i}\right) .
$$

We denote $C_{r}^{p}=\left\{(p, q) \in Q^{2 n+1} \times Q^{2 n}: p_{1}=0, \ldots, p_{r}=0\right\} \quad r \geq$ 2. By reduction $\pi_{H}: C \cap C_{r}^{p} \rightarrow X$ we obtain the corresponding coisotropic varieties in the space of pairs of polynomials with roots of multiplicity greater than or equal to $(0, r-1)$. Their generating families can be written as

$$
G_{r}(\bar{q}, \alpha, \lambda)=\sum_{i=r+1}^{2 n+1} \lambda_{i}\left(\alpha_{i}-\sum_{l=2}^{i} \bar{q}_{l-1} \frac{1}{(i-l) !} \lambda^{i-l}-\frac{1}{i !} \lambda^{i}\right) .
$$

In an analogous way we obtain the generating families for coisotropic varieties $\pi_{H}\left(C \cap C_{s}^{q}\right), C_{s}^{q}=\left\{(p, q) \in Q^{2 n+1} \times Q^{2 n}: q_{2 n+1}=0, \ldots\right.$, $\left.q_{2 n+2-s}=0\right\}, s \geq 2$, namely

$$
\begin{aligned}
H_{s}(\bar{q}, \alpha, \lambda)= & \sum_{i=2}^{2 n+1-s} \lambda_{i}\left(\alpha_{i}-\sum_{l=2}^{i} \bar{q}_{l-1} \frac{1}{(i-l) !} \alpha_{1}^{i-l}-\frac{1}{i !} \alpha_{1}^{i}\right) \\
& -\sum_{r=2 n+2-s}^{2 n+1} \lambda_{r}\left(\sum_{l=2}^{r} \bar{q}_{l-1} \frac{1}{(r-l) !} \alpha_{1}^{r-l}+\frac{1}{r !} \alpha_{1}^{r}\right) .
\end{aligned}
$$

We see that $\pi_{H}\left(C \cap C_{r}^{p} \cap C_{s}^{q}\right) \subset X$ form the lagrangian varieties. The irreducible component of the case $r=n+1, s=n$ is known as the open Whitney umbrella singular lagrangian variety (see [Giv], [Ar2]), which appeared as a generic singularity of systems of rays passing through the singular initial conditions represented by the generic isotropic submanifolds situated in given hypersurface $\{H=0\}$.

By straightforward calculations using the generating family $F$ for $C$, we obtain the generating family for lagrangian variety 
$\pi_{H}\left(C \cap C_{n+1}^{p} \cap C_{n}^{q}\right)$, namely

$$
\begin{aligned}
W(\bar{q}, \bar{\lambda})= & \int_{0}^{\lambda}\left(\sum_{l=2}^{n+1} \frac{1}{(n+1-l) !} t^{n+1-l} \bar{q}_{l-1}+\frac{1}{(n+1) !} t^{n+1}\right) \\
& \times\left(\sum_{i=0}^{n-1}(-1)^{i} \lambda_{i+1} \frac{1}{i !} t^{i}\right) d t+\sum_{i=0}^{n-1} \lambda_{i+1} \bar{q}_{i+n+1}, \\
\bar{\lambda}= & \left(\lambda, \lambda_{1}, \ldots, \lambda_{n}\right) .
\end{aligned}
$$

This variety has two components: the first one forms the pairs of $(F, G)$ with roots of multiplicity at least $(n+1, n)$. The second forms the pairs $(F, G)$ with roots of multiplicity at least $(n, n+1)$. The first component is called an open Whitney umbrella and is generated by the degenerate $I$-generating family:

$$
\begin{aligned}
F_{1}(\bar{q}, \beta, \bar{\lambda})= & \lambda_{1}\left(\beta-\sum_{l=2}^{n+1} \frac{1}{(n+1-l) !} \lambda^{n+1-l} \bar{q}_{l-1}-\frac{1}{(n+1) !} i \lambda^{n+1}\right) \\
& -\sum_{i=2}^{n+1} \lambda_{i}\left(\sum_{l=2}^{n+i} \frac{1}{(n+i-l) !} \lambda^{n+i-l} \bar{q}_{l-1}+\frac{1}{(n+i) !} \lambda^{n+i}\right), \\
\bar{\lambda}= & \left(\lambda, \lambda_{1}, \ldots, \lambda_{n+1}\right) .
\end{aligned}
$$

Intersection of both components is an isotropic variety (polynomials with roots of multiplicity at least $(n+1, n+1))$, with the generating family

$$
\begin{aligned}
F_{2}(\bar{q}, \beta, \bar{\lambda})= & \sum_{k=1}^{2} \lambda_{k}\left(\beta_{k}-\sum_{l=2}^{n+k} \frac{1}{(n+k-l) !} \lambda^{n+k-l} \bar{q}_{l-1}-\frac{1}{(n+k) !} \lambda^{n+k}\right) \\
& -\sum_{i=3}^{n+1} \lambda_{i}\left(\sum_{l=2}^{n+i} \frac{1}{(n+i-l) !} \lambda^{n+i-l} \bar{q}_{l-1}+\frac{1}{(n+i) !} \lambda^{n+i}\right), \\
\beta= & \left(\beta_{1}, \beta_{2}\right), \quad \bar{\lambda}=\left(\lambda, \lambda_{1}, \ldots, \lambda_{n+1}\right) .
\end{aligned}
$$

2. Classification of isotropic submanifolds. Let $(I, 0)$ be a germ of a proper isotropic submanifold of $\left(T^{*} M, \omega_{M}\right)$. Let

$$
G:\left(M \times R^{L} \times R^{K},(0,0,0)\right) \rightarrow R
$$

be a corresponding germ of the $I$-Morse family generating $(I, 0)$.

Finding the generating families for coisotropic and isotropic submanifolds suggests the corresponding groups of equivalences of unfoldings preserving the fiber structure given on the space of unfolding 
parameters (cf. [Was]). In contrast the natural group of equivalences for $I$-generating families is formed by diffeomorphisms preserving the corner in the space of Morse parameters. In singularity theory that group was first introduced by Arnold [AVG], then generalized by Siersma [Sie], and first applied in symplectic geometry of holonomic differential systems by Pham [Pha].

Let $\mathscr{E}_{(q, \beta, \lambda)}$ denote the space of smooth function germs at zero defined on $M \times R^{L} \times R^{K}$. By $\mathscr{M}_{(q, \beta, \lambda)}$ we denote the maximal ideal of $\mathscr{E}_{(q, \beta, \lambda)}$. By $\left\langle\beta_{1}, \ldots, \beta_{L}\right\rangle \mathscr{E}_{(q, \beta, \lambda)}$ we denote the ideal of $\mathscr{E}_{(q, \beta, \lambda)}$ generated by $\beta_{1}, \ldots, \beta_{L}$ coordinate functions.

Let $\mathscr{D}(L, K)$ be the set of germs at $(0,0)$ of diffeomorphisms of $\left(R^{L} \times R^{K},(0,0)\right)$ preserving the hyperplanes $\left\{(\beta, \lambda) \in R^{L} \times R^{K}\right.$; $\left.\beta_{i}=0\right\}$ and the $L$-dimensional corner $\mathscr{H}=\left\{(\beta) \in R^{L} ; \beta_{i} \geq 0\right.$, $i=1, \ldots, L\}$ in $R^{L} \times R^{K}$ (cf. [Sie]).

Definition 2.1. Two I-generating families (germs),

$$
G_{1,2}:\left(M \times R^{L} \times R^{K},(0,0,0)\right) \rightarrow R
$$

are said to be $I$-equivalent if there exist the germ of diffeomorphism $\Phi:\left(M \times R^{L} \times R^{K}, 0\right) \rightarrow\left(M \times R^{L} \times R^{K}, 0\right), \quad \Phi(q, \cdot, \cdot) \in \mathscr{D}(L, K)$, and a smooth function-germ $\alpha \in\left\langle\beta_{1}, \ldots, \beta_{L}\right\rangle^{2} \mathscr{E}_{(q, \beta, \lambda)}$ such that the following diagram commutes

$$
\begin{aligned}
& \left(M \times R^{L} \times R^{K}, 0\right) \stackrel{\Phi}{\rightarrow}\left(M \times R^{L} \times R^{K}, 0\right) \\
& \pi_{M} \searrow \pi_{M}
\end{aligned}
$$

and

$$
G_{1} \circ \Phi+\alpha=G_{2} .
$$

We easily see that the corresponding isotropic submanifolds defined in (2) by $I$-equivalent $I$-Morse families are identical. Now we use the standard group of symplectic equivalences (cf. [AVG]).

Definition 2.2. We say that two germs of isotropic varieties $\left(I_{1}, 0\right),\left(I_{2}, 0\right) \subset\left(T^{*} M, \omega_{M}\right)$ are equivalent if there exists a symplectomorphism $\Phi: T^{*} M \rightarrow T^{*} M$ preserving the fiber bundle structure such that

$$
\Phi\left(I_{1}\right) \subset I_{2}, \quad \Phi(0)=0 .
$$

We see that two $I$-Morse families $G_{1}, G_{2}$ generate two equivalent isotropic submanifolds $I_{1}, I_{2}$, if and only if there exist a diffeomor- 
phism $\varphi:(M, 0) \rightarrow(M, 0)$ and a smooth function-germ $g:(M, 0)$ $\rightarrow(R, 0)$ such that $G_{1} \circ(\varphi$, id $)+g \circ \pi_{M}$ and $G_{2}$ are $I$-equivalent. In this case $G_{1}, G_{2}$ are said to be equivalent.

Now following the standard lines of singularity theory of functions on boundaries and corners (cf. [AVG], [Sie]), we classify the versal isotropic submanifolds of $\left(T^{*} M, \omega_{M}\right)$ by classification of the corresponding versal $I$-generating families.

Let $\mathscr{E}_{(\beta, \lambda)}$ denote the space of smooth function-germs at zero defined on $R^{L} \times R^{K}$. Let $f \in \mathscr{E}_{(\beta, \lambda)}, f(0)=0$. An $n$-dimensional unfolding of $f$ is a germ $F \in \mathscr{E}_{(x, \beta, \lambda)}$ such that $\left.F\right|_{\{0\} \times R^{L} \times R^{K}}=f$. An unfolding $F$ of $f$ is called versal if given any other unfolding $H \in \mathscr{E}(y, \beta, \lambda)$ of $f$ there is a triple $(\Phi, \alpha, g)$, where

(i) $\Phi(x, \beta, \lambda)=(\psi(x), \varphi(x, \beta, \lambda)) \in R^{m} \times R^{L} \times R^{k},\left.\varphi\right|_{\{0\} \times R^{L} \times R^{K}}$ $=\operatorname{id}_{R^{L} \times R^{K}}, \varphi(0)=0$,

(ii) $\alpha \in\left\langle\beta_{1}, \ldots, \beta_{L}\right\rangle \mathscr{E}_{(x, \beta, \lambda)}, g \in \mathscr{E}_{(x)}$,

(iii) for $(x, \beta, \lambda) \in R^{n} \times R^{L} \times R^{K}$, we have

$$
F(x, \beta, \lambda)=H(\Phi(x, \beta, \lambda))+\alpha(x, \beta, \lambda)+g(x) .
$$

By $\Delta_{L, K}(f)$ we denote the Jacobi ideal of $f \in \mathscr{E}_{(\beta, \lambda)}$, namely

$$
\Delta_{L, K}(f)=\left\langle\beta_{1} \frac{\partial f}{\partial \beta_{1}}, \ldots, \beta_{L} \frac{\partial f}{\partial \beta_{L}}, \frac{\partial f}{\partial \lambda_{1}}, \ldots, \frac{\partial f}{\partial \lambda_{K}}\right\rangle \mathscr{E}_{(\beta, \lambda)} .
$$

We say that $f \in \mathscr{E}_{(\beta, \lambda)}$ has finite codimension $c$ (equivalently we say $f$ is finitely determined [Sie]) if

$$
\operatorname{cod}(f)=c=\operatorname{dim}_{R} \frac{\mathscr{M}_{(\beta, \lambda)}}{\Delta_{L, K}(f)+\left\langle\beta_{1}, \ldots, \beta_{L}\right\rangle^{2} \mathscr{E}_{(\beta, \lambda)}}<\infty .
$$

If $c$ is finite then $c$ is the minimal dimension of a versal unfolding of $f$. If $g_{1}, \ldots, g_{c} \in \mathscr{M}_{(\beta, \lambda)}$ are representatives of a basis of

$$
\frac{\mathscr{M}_{(\beta, \lambda)}}{\Delta_{L, K}(f)+\left\langle\beta_{1}, \ldots, \beta_{L}\right\rangle^{2 \mathscr{E}}(\beta, \lambda)}
$$

then the miniversal unfolding of $f$ is following (cf. [Mar])

$$
H(x, \beta, \lambda)=f(\beta, \lambda)+\sum_{i=1}^{c} x_{i} g_{i}(\beta, \lambda) .
$$

Definition 2.3. Let $(x, \beta, \lambda) \rightarrow G(x, \beta, \lambda)$ be an $I$-generating family for a germ of the isotropic submanifold $(I, 0) \subset T^{*} M$. We say that $(I, 0)$ is versal if $G$ is versal.

Versality assures that all isotropic germs with fixed finite-determined $G(0, \beta, \lambda)$ are generated by an $I$-generating family (not necessarily 
$I$-Morse family) obtained by an arbitrary pullback from the versal unfolding of $G(0, \beta, \lambda)$ (cf. [Mar]).

To classify $(I, 0)$-versal isotropic submanifolds we need at first to classify singularities of $f \in \mathscr{M}_{(\beta, \lambda)}^{2}$ with respect to the modified $\mathscr{D}_{L, K^{-}}$ equivalence; i.e. we say that $f, f^{\prime} \in \mathscr{M}_{(\beta, \lambda)}^{2}$ are equivalent if there is a function $g \in\left\langle\beta_{1}, \ldots, \beta_{L}\right\rangle^{2} \mathscr{E}_{(\beta, \lambda)}$ such that $f$ and $f^{\prime}+g$ are $\mathscr{D}_{L, K}$-right equivalent.

LEMMA 2.4 (on the pre-normal form). Let $f \in \mathscr{M}_{(\beta, \lambda)}^{2}$. Then there are nonnegative numbers $k, K_{1}$, linear functions $l_{i}$ of $\bar{\lambda}=\left(\lambda_{1}, \ldots, \lambda_{k}\right)$ variables, $1 \leq i \leq L-k$, smooth functions $\varphi_{i}$ in $\mathscr{M}_{\left(K_{1}\right)}^{2}, \psi \in \mathscr{M}_{\left(K_{1}\right)}^{3}$ such that $f$ is equivalent to

$$
\begin{aligned}
\beta_{1} \lambda_{1}+\cdots+\beta_{k} \lambda_{k}+\sum_{i=1}^{L-k} \beta_{k+i}\left(l_{i}(\bar{\lambda})+\varphi_{i}(\lambda)\right) \\
+\psi(\lambda)+Q\left(\lambda_{K_{1}+1}, \ldots, \lambda_{K}\right),
\end{aligned}
$$

where $k \leq K_{1} \leq K$ and $Q$ is a nondegenerate quadratic form of $\lambda_{K_{1}+1}, \ldots, \lambda_{K}$-variables.

Proof. Using the splitting lemma (see [Sie], p. 122) and reducing the terms of order two or higher in $(\beta)$ we find the prenormal form (3).

By this lemma and using the determinacy criteria (cf. [Ja1]) we have the following classification theorem (cf. [Mat] in ordinary case).

Proposition 2.5. A. We assume $L=1$ and the corank of $f$ is 2. Then there are two series of simple singularities with the following versal unfoldings:

$$
\begin{aligned}
& K_{p}^{q}: \quad \beta \lambda^{q}+\lambda^{p}+\sum_{j=1}^{q-1} x_{j} \beta \lambda^{j-1}+\sum_{i=1}^{p-1} x_{q+i-1} \lambda^{i}, \\
& q \geq 1, p \geq 3, c=q+p-2, \\
& F_{s}: \quad \lambda^{s-1}+\sum_{j=0}^{s-3} x_{s+j-2} \beta \lambda^{j}+\sum_{i=1}^{s-3} x_{i} \lambda^{i}, \quad s \geq 3, c=2 s-5 .
\end{aligned}
$$

B. Unimodal, corank 3 (corank $f=L+K$ ) singularities with $L=1$ 
are equivalent to one from the following list,

$$
\begin{array}{rll}
L_{6}: & \lambda_{1}^{2} \lambda_{2} \pm \lambda_{2}^{3}+\beta \lambda_{1}+a \beta \lambda_{2}, & c=5, a \neq 1, \\
D_{k, l}: & \lambda_{1}^{2} \lambda_{2} \pm \lambda_{2}^{k-1}+\beta \lambda_{2}+a \beta \lambda_{1}^{l}, & c=k+l, a \neq 0, \\
& & k+l>5, k \geq 4, \\
E_{6,0}: & \lambda_{1}^{3} \pm \lambda_{2}^{4}+\beta \lambda_{2}+a \beta \lambda_{1}, & c=7, \\
E_{7,0}: & \lambda_{1}^{3}+\lambda_{1} \lambda_{2}^{3}+\beta \lambda_{2}+a \beta \lambda_{1}, & c=8, \\
E_{8,0}: & \lambda_{1}^{3}+\lambda_{2}^{5}+\beta \lambda_{2}+a \beta \lambda_{1}, & c=9, \\
D_{5}^{1}: & \lambda_{1}^{2} \lambda_{2} \pm \lambda_{2}^{4}+\beta \lambda_{1}+a \beta \lambda_{2}^{2}, & c=7, \\
E_{6,1}: & \lambda_{1}^{3} \pm \lambda_{2}^{4}+\beta \lambda_{1}+a \beta \lambda_{2}^{2}, & c=8 .
\end{array}
$$

Since now we assume that our $I$-generating family has a minimal number of $\lambda$-parameters, i.e. $\left(\partial^{2} f / \partial \lambda_{i} \partial \lambda_{j}\right)(0)=0$. Changing the $I$ generating family by subtracting or adding nondegenerate quadratic forms of the remaining variables is called a stable equivalence of generating families (cf. [AVG]).

3. Quasicaustics and their evolutions. Let $(I, 0)$ be an isotropic submanifold of $\left(T^{*} M, \omega_{M}\right)$ with $\operatorname{codim} I=\operatorname{dim} M+r, r \geq 1$.

Definition 3.1. We call the image set $\left(\pi_{M}(I), 0\right) \subset M$ a quasicaustic corresponding to $(I, 0)$.

Let $(q, \beta, \lambda) \rightarrow G(q, \beta, \lambda)$ be a generating family for $(I, 0)$. We see that the quasicaustic of $(I, 0)$ can be written in the following way,

$$
Q(I)=\left\{q \in M: G(q, \cdot, \cdot) \text { has a critical point on }\{0\} \times R^{K}\right\} .
$$

If $\pi_{1}: M \times R^{L} \times R^{K} \rightarrow M$ denotes the natural projection on the first factor then we can write

$$
Q(I)=\pi_{1}\left(\Sigma_{G}^{I}\right)
$$

The notion of quasicaustic was introduced in [Ja2] in the context of geometric optics of diffraction on apertures (cf. Example 1.4). Generalization to the system of apertures was given in [JP].

On the basis of the preceding section (Proposition 2.5), by straightforward checking, we obtain

Proposition 3.2. The only stable quasicaustics for $L=1$ in dimension $\leq 4$ are diffeomorphic to hypersurface or Whitney's cross cap $Q\left(F_{4}\right)$, extended by a cartesian product with Euclidean space. 
To study the evolution of quasicaustics we follow Arnol'd [Ar2]; we first study the singularities of functions on quasicaustics. To determine the normal forms of these functions we need to compute the module of logarithmic vector fields tangent to the given quasicaustic (cf. $[\mathbf{J P}]$ ).

Let $(I, 0)$ be an isotropic, versal germ. Let

$$
G(q, \beta, \lambda)=f(\beta, \lambda)+\sum_{i=1}^{c} q_{i} g_{i}(\beta, \lambda)
$$

be its versal $I$-generating family. We restrict our considerations to the complex analytic or real analytic case, $\mathscr{O}_{(q)}, \mathscr{O}_{(q, \beta, \lambda)}$ etc. By Derlog $Q(I)$ we denote $\mathscr{O}_{(q)}$-module of germs of analytic vector fields on $\left(R^{n}, 0\right)$ which are tangent to the nonsingular part of $Q(I)$.

Using the Malgrange Preparation Theorem (cf. [Mar]) we see that the module

$$
\mathscr{O}_{(q, \beta, \lambda)} / \bar{\Delta}_{L, K}(G)
$$

where

$$
\bar{\Delta}_{L, K}(G)=\left\langle\beta_{l} \frac{\partial G}{\partial \beta_{l}}, \frac{\partial G}{\partial \lambda_{k}}, \beta_{i} \beta_{j}\right\rangle \mathscr{O}_{(q, \beta, \lambda)},
$$

$1 \leq l \leq L, 1 \leq k \leq K, 1 \leq i, j \leq L$, is a free $\mathscr{O}_{(q)}$-module generated by $1, g_{1}, \ldots, g_{c}$. Thus for each $h \in \mathscr{O}_{(q, \beta, \lambda)}$ we have

$$
\begin{aligned}
h(q, \beta, \lambda)= & \sum_{l=1}^{L} \gamma_{l} \beta_{l} \frac{\partial G}{\partial \beta_{l}}+\sum_{k=1}^{K} \delta_{k} \frac{\partial G}{\partial \lambda_{k}} \\
& +\sum_{i=1}^{c} \alpha_{i} g_{i}+\alpha+\sum_{i \leq j=1}^{L} \beta_{i} \beta_{j} \xi_{i, j},
\end{aligned}
$$

where $\gamma_{l}, \delta_{k}, \xi_{i, j} \in \mathscr{O}_{(q, \beta, \lambda)}$ and $\alpha, \alpha_{i} \in \mathscr{O}_{(q)}$. If $h \in \mathscr{O}_{(q, \beta, \lambda)}$ satisfies the conditions

$$
\frac{\partial h}{\partial \lambda_{k}}(\cdot, 0, \cdot), \quad \frac{\partial h}{\partial \beta_{l}}(\cdot, 0, \cdot) \in\left\langle\frac{\partial G}{\partial \beta_{l}}(\cdot, 0, \cdot), \frac{\partial G}{\partial \lambda_{k}}(\cdot, 0 ., \cdot)\right\rangle,
$$

then the vector field $\eta=\sum_{i=1}^{n} \alpha_{i}\left(\partial / \partial q_{i}\right)$, where $\alpha_{c+1}, \ldots, \alpha_{n}$ are arbitrary elements of $\mathscr{O}_{(q)}$, is tangent to $Q(I)$ (cf. [JP]).

Let $Q(I)$ be a quasicaustic and $g:\left(R^{n} \supset Q(I), 0\right) \rightarrow R$ be a smooth function. We wish to classify these functions up to changes of coordinates in $R^{n}$ which preserve the quasicaustic $Q(I)$. These we obtain by integrating vector fields obtained from the basis, say $\vartheta_{i}$, constructed above. We denote the resulting group of diffeomorphisms 
by $\mathscr{G}(Q(I))$. Using the standard results of singularity theory on varieties we obtain the analogous notions of stability and determinacy (see [Bru], [AVG]).

We use the Jacobi ideal $\left\langle\vartheta_{1} g, \ldots, \vartheta_{s} g\right\rangle \mathscr{O}_{n}$. Thus we have the criterion for stability of $g$, namely $g$ is $\mathscr{G}(Q(I))$-stable if and only if

$$
\mathscr{M}_{n}^{2}+\left\langle\vartheta_{1} g, \ldots, \vartheta_{s} g\right\rangle \mathscr{O}_{n} \supset \mathscr{M}_{n},
$$

where $\mathscr{M}_{n}$ is a maximal ideal in $\mathscr{O}_{(q)}$. Thus if we have a family of functions on $Q(I)$, say $W:\left(R^{n} \times U, 0 \times U\right) \rightarrow(R, 0)$ parametrized by an open set $U \subset R^{p}$ and $W_{u}(\cdot)=W(\cdot, u)$ is stable for each $u \in U$. Then if $u_{1}, u_{2}$ are in the same component of $U$ stability implies that $W_{u_{1}}$ and $W_{u_{2}}$ are $\mathscr{G}(Q(I))$-equivalent provided all $\vartheta_{i}$ vanish at $0 \in Q(I)$. Now we have the following

Proposition 3.3. Let $\left(Q\left(F_{4}\right), 0\right)$ be the stable quasicaustic in $R^{3}$ corresponding to an $F_{4}$-singularity. Then under the generic condition

$$
\frac{\partial g}{\partial q_{1}}(0) \frac{\partial g}{\partial q_{3}}(0) \neq 0
$$

the function $g$ on $Q\left(F_{4}\right)$ is stable and $\mathscr{G}\left(Q\left(F_{4}\right)\right)$-equivalent to one of the functions,

$$
g(q)= \pm q_{1} \pm q_{3}
$$

Proof. At first we show that $g$ is a stable germ. By straightforward calculations using formula (4) we find the generators of the $\mathscr{O}_{(q)}$ module of logarithmic vector fields Derlog $Q\left(F_{4}\right)$ (cf. [Ja2]), namely:

$$
\begin{array}{ll}
\vartheta_{1}=-\frac{1}{6} q_{3}^{2} \frac{\partial}{\partial q_{2}}+q_{2} \frac{\partial}{\partial q_{1}}, & \vartheta_{2}=q_{3} \frac{\partial}{\partial q_{3}}+q_{2} \frac{\partial}{\partial q_{2}} \\
\vartheta_{3}=-\frac{1}{3} q_{3} \frac{\partial}{\partial q_{3}}+\frac{2}{3} q_{1} \frac{\partial}{\partial q_{1}}, & \vartheta_{4}=q_{2} \frac{\partial}{\partial q_{3}}-\frac{1}{3} q_{3} q_{1} \frac{\partial}{\partial q_{2}} .
\end{array}
$$

So we have

$$
\begin{aligned}
\mathscr{M}_{(q)}^{2}+\left\langle\vartheta_{i}(g)\right\rangle= & \left\langle-\frac{1}{6} q_{3}^{2} \frac{\partial g}{\partial q_{2}}+q_{2} \frac{\partial g}{\partial q_{1}}, q_{3} \frac{\partial g}{\partial q_{3}}+q_{2} \frac{\partial g}{\partial q_{2}},\right. \\
& \left.\quad-\frac{1}{3} q_{3} \frac{\partial g}{\partial q_{3}}+\frac{2}{3} q_{1} \frac{\partial g}{\partial q_{1}}, q_{2} \frac{\partial g}{\partial q_{3}}-\frac{1}{3} q_{3} q_{1} \frac{\partial g}{\partial q_{2}}\right\rangle \\
\supseteq \mathscr{M}_{(q)} \mathscr{O}_{(q)} . &
\end{aligned}
$$

This implies that $g$ is 1 -determined by its one-jet $j^{1} g$. Let $g(q)=$ $a_{1} q_{3}+a_{2} q_{2}+a_{3} q_{1}$ so we obtain the four types of stable functions defined by the connected components of the complement of the variety 
a)

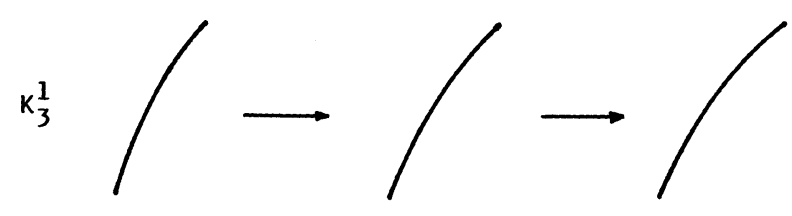

b)

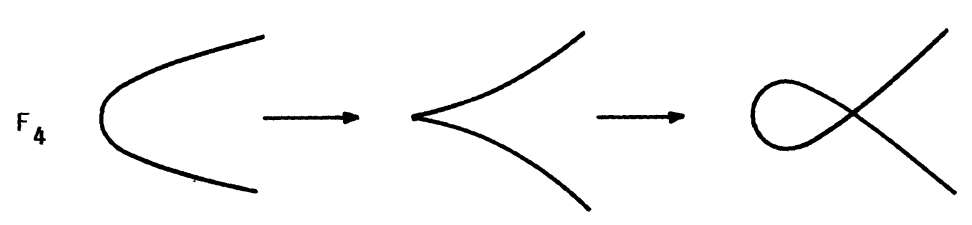

Figure 1

$\left\{a_{1} a_{3}=0\right\}$. Therefore by local triviality property of $g$ we obtain the desired result.

Corollary 3.4. A generic (and stable) function on $Q\left(F_{4}\right) \times R^{k}$ is equivalent to

1. $y_{1}$, or

2. $\pm q_{1} \pm q_{3} \pm y_{1}^{2} \pm \cdots \pm y_{k}^{2}$, where $\left\{y_{i}\right\}_{1}^{k}$ are coordinates on $R^{k}$.

By simple checking of the intersection of the "big" caustic $Q\left(F_{4}\right)$ with the family of the level sets of the functions $\pm q_{1} \pm q_{3}$ we obtain the following result.

COROLlaRY 3.5. Generic evolution of quasicaustics in the plane are illustrated in Figure 1 a, b.

Using the stable families of level sets defined by functions of Corollary 3.4 , namely:

$$
t=y_{1}, \quad t= \pm q_{1} \pm q_{3} \pm y_{1}^{2}
$$

on quasicaustic $Q\left(F_{4}\right) \times R$ we obtain the following result.

COROLlary 3.6. Generic evolution of quasicaustics in $R^{3}$ are illustrated in Figure $2 \mathrm{a}, \mathrm{b}, \mathrm{c}, \mathrm{d}(L=1)$. 
a)

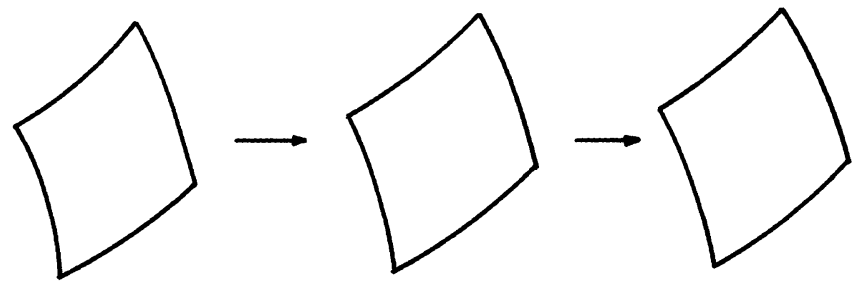

b)
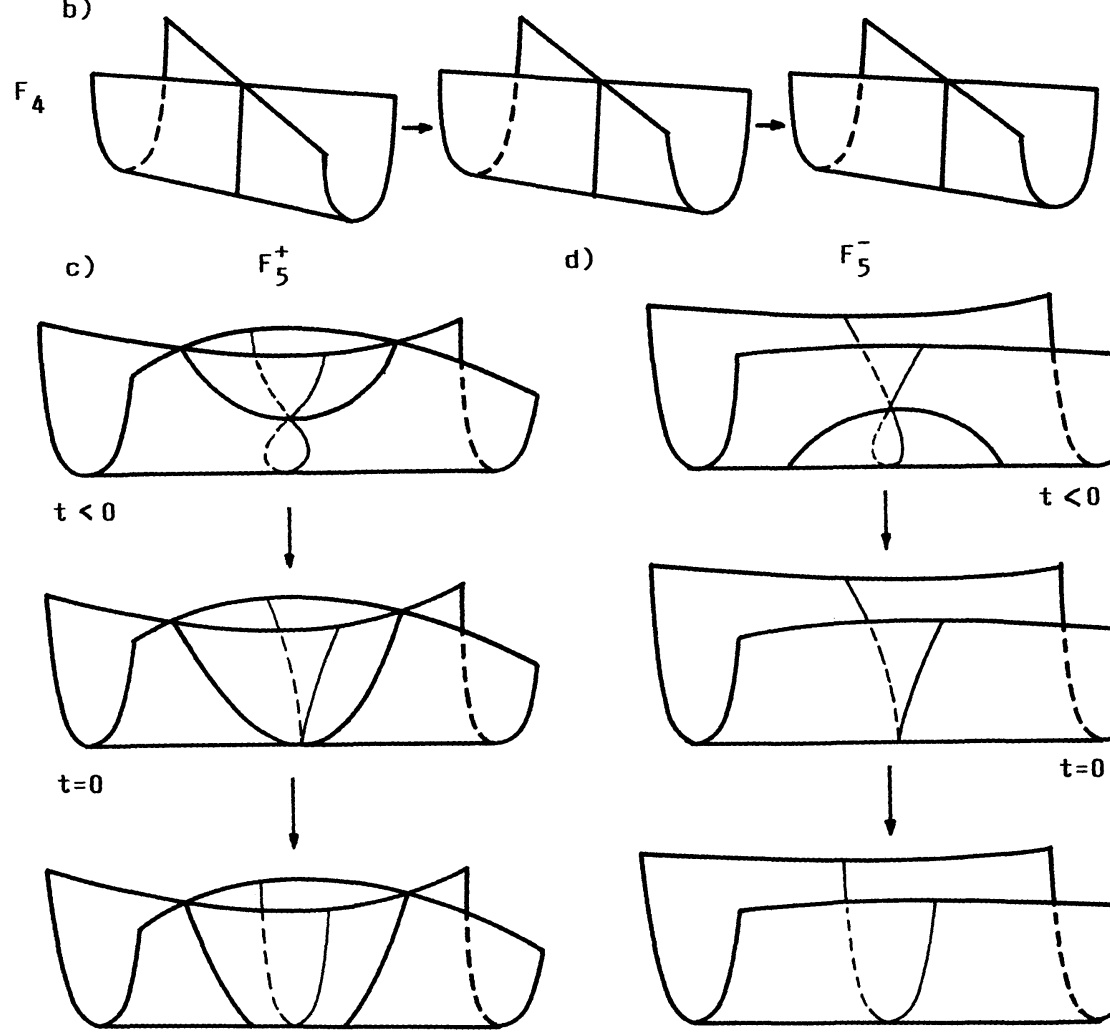

$t>0$
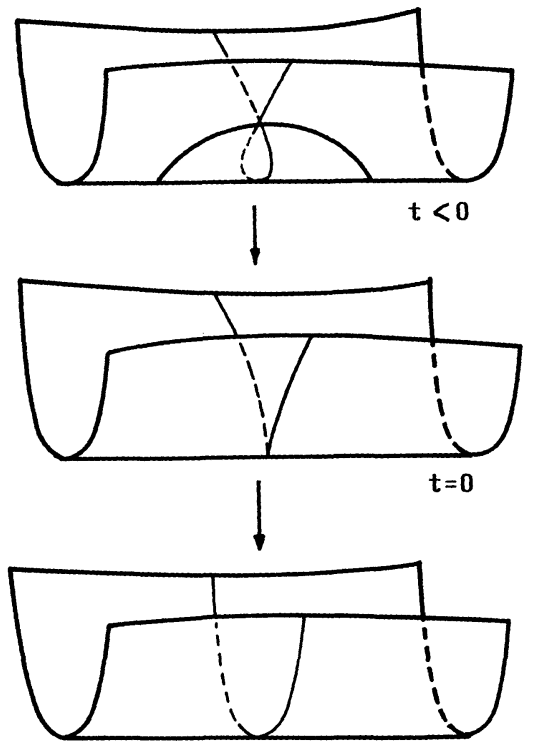

$t>0$

FIGURE 2

\section{REFERENCES}

[AM] R. Abraham and J. E. Marsden, Foundations of Mechanics, (2nd ed.), Benjamin (Cummings, Reading), 1978.

[AGV] V. I. Arnold, S. M. Gusein-Zade and A. N. Varchenko, Singularities of Differentiable Maps, Vol. 1, Birkhäuser, Boston, 1985, (English ed.).

[Ar1] V. I. Arnold, Singularities in variational calculus, Itogi Nauki, Contemporary Problems in Mathematics, 22 (1983), 3-55, (Russian ed.).

[Ar2] _ Evolution of singularities of potential flows in collisions free media and caustic metamorphosae in 3-space, Proc. of The Petrovskij Seminar, 8 (1982), 21-57. 
[Br] J. W. Bruce, Functions on discriminants, J. London Math. Soc., (2), 30 (1984), 551-567.

[Dui] J. Duistermaat, Oscilatory integrals, Lagrange immersions and unfoldings of singularities, Comm. Pure Appl. Math., 27 (1974), 207-281.

[Giv] A. B. Givental, Singular Lagrangian manifolds and their Lagrange projections, Itogi Nauki, Contemporary Problems in Mathematics, 33 (1988), 55112.

[Hör] L. Hörmander, Fourier integral operators I, Acta Math., 127 (1971), 79-183.

[Ja1] S. Janeczko, On algebraic criteria for $k$-determinacy of germs of smooth functions on manifolds with boundary, Demonstratio Math., 15, No. 4 (1982), 1113-1123.

[Ja2] _ Generalized Luneburg canonical varieties and vector fields on quasicaustics, J. Math. Phys., 31, No. 4 (1990), 997-1009.

[Ja3] _ Coisotropic varieties and their generating families, Ann. Inst. H. Poincaré, Phys. Théorique, 56, no. 4, (1992), 429-441.

[JP] S. Janeczko and G. Plotnikova, Sur la structure de quasicaustiques en diffraction géométrique, C. R. Sci. Paris t.311, Serie I (1990), 543-546.

[JR] S. Janeczko and R. M. Roberts, Classification of symmetric caustics I: Symplectic equivalence, Proceedings of symposium on singularity theory and its applications, Univ. of Warwick 1989, vol. 2: Singularities, bifurcations, and dynamics, Springer Lecture Notes in Mathematics, 1463 (1991), 193-213.

[Kel] J. B. Keller, Rays, waves and asymptotics, Bull. Amer. Math. Soc., 84 (1978), 727-749.

[KR] J. P. S. Kung and G.-C. Rota, Invariant theory of binary forms, Bull. Amer. Math. Soc., 10 (1984), 27-85.

[Mar] J. Martinet, Singularities of Smooth Functions and Maps, Cambridge Univ. Press, Cambridge 1982.

[Mat] V. I. Matov, Unimodal and bimodal germs of functions on manifold with boundary, Proc. Petrovski Sem., 7 (1981), 174.

[Pha] F. Pham, Singularités des systemes différentiels de Gauss-Manin, Progress in Math. 2, Birkhäuser 1979.

[Sie] D. Siersma, Singularities of functions on boundaries, corners, etc., Quart. J. Math. Oxford (2), 32 (1981), 119.

[Wal] C. T. C. Wall, Geometric properties of generic differentiable manifolds, in Geometry and Topology, Springer Lecture Notes in Mathematics, vol. 597, Springer, Berlin, (1977), 707-774.

[Was] G. Wassermann, Stability of unfoldings in space and time, Acta Math., 135 (1975), 58-128.

[Wei] A. Weinstein, Lectures on symplectic manifolds, C.B.M.S. Conf. Series, Amer. Math. Soc. 29 (1977).

[Zak] V. Zakalyukin, The generalization of lagrangian triads, Uspekhi Mat. Nauk, 41, No. 4 (1986), 180.

Received July 1, 1991 and in revised form February 13, 1992.

\section{TECHNICAL UNIVERSITY OF WARSAW}

Pl. Politechniki 1

00-661 Warsaw, Poland 


\title{
PACIFIC JOURNAL OF MATHEMATICS \\ Founded by \\ E. F. BECKENBACH (1906-1982) F. WolF (1904-1989)
}

\section{EDITORS}

\section{S. VARADARAJAN \\ (Managing Editor) \\ University of California \\ Los Angeles, CA 90024-1555 \\ vsv@math.ucla.edu}

\section{F. Michael Christ}

University of California

Los Angeles, CA 90024-1555

christ@math.ucla.edu

\section{Herbert Clemens}

University of Utah

Salt Lake City, UT 84112

clemens@math.utah.edu

\author{
THOMAs ENRIGHT \\ University of California, San Diego \\ La Jolla, CA 92093 \\ tenright@ucsd.edu \\ Nicholas ERcolani \\ University of Arizona \\ Tucson, AZ 85721 \\ ercolani@math.arizona.edu \\ R. FINN \\ Stanford University \\ Stanford, CA 94305 \\ finn@gauss.stanford.edu \\ VAUghan F. R. JoNeS \\ University of California \\ Berkeley, CA 94720 \\ vfr@math.berkeley.edu
}

\section{SUPPORTING INSTITUTIONS}

UNIVERSITY OF ARIZONA

UNIVERSITY OF BRITISH COLUMBIA

CALIFORNIA INSTITUTE OF TECHNOLOGY

UNIVERSITY OF CALIFORNIA

UNIVERSITY OF MONTANA

UNIVERSITY OF NEVADA, RENO

NEW MEXICO STATE UNIVERSITY OREGON STATE UNIVERSITY

\author{
UNIVERSITY OF OREGON \\ UNIVERSITY OF SOUTHERN CALIFORNIA \\ STANFORD UNIVERSITY \\ UNIVERSITY OF HAWAII \\ UNIVERSITY OF UTAH \\ WASHINGTON STATE UNIVERSITY \\ UNIVERSITY OF WASHINGTON
}

The Supporting Institutions listed above contribute to the cost of publication of this Journal, but they are not owners or publishers and have no responsibility for its content or policies.

Mathematical papers intended for publication in the Pacific Journal of Mathematics should be in typed form or offset-reproduced (not dittoed), double spaced with large margins. Please do not use built up fractions in the text of the manuscript. However, you may use them in the displayed equations. Underline Greek letters in red, German in green, and script in blue. The first paragraph must be capable of being used separately as a synopsis of the entire paper. In particular it should contain no bibliographic references. Please propose a heading for the odd numbered pages of less than 35 characters. Manuscripts, in triplicate, may be sent to any one of the editors. Please classify according to the 1991 Mathematics Subject Classification scheme which can be found in the December index volumes of Mathematical Reviews. Supply name and address of author to whom proofs should be sent. All other communications should be addressed to the managing editor, or Julie Speckart, University of California, Los Angeles, California 90024-1555.

There are page-charges associated with articles appearing in the Pacific Journal of Mathematics. These charges are expected to be paid by the author's University, Government Agency or Company. If the author or authors do not have access to such Institutional support these charges are waived. Single authors will receive 75 free reprints; joint authors will receive a total of 100 free reprints. Additional copies may be obtained at cost in multiples of 50 .

The Pacific Journal of Mathematics (ISSN 0030-8730) is published monthly except for July and August. Regular subscription rate: $\$ 200.00$ a year (10 issues). Special rate: $\$ 100.00$ a year to individual members of supporting institutions.

Subscriptions, orders for numbers issued in the last three calendar years, and changes of address should be sent to Pacific Journal of Mathematics, P.O. Box 969, Carmel Valley, CA 93924, U.S.A. Old back numbers obtainable from Kraus Periodicals Co., Route 100, Millwood, NY 10546.

The Pacific Journal of Mathematics at P.O. Box 969, Carmel Valley, CA 93924 (ISSN 0030-8730) is published monthly except for July and August. Second-class postage paid at Carmel Valley, California 93924, and additional mailing offices. Postmaster: send address changes to Pacific Journal of Mathematics, P.O. Box 969, Carmel Valley, CA 93924.

PUBLISHED BY PACIFIC JOURNAL OF MATHEMATICS, A NON-PROFIT CORPORATION

This publication was typeset using $\mathcal{A} \mathcal{S} S-\mathrm{T}_{\mathrm{E}} \mathrm{X}$, the American Mathematical Society's $\mathrm{T}_{\mathrm{E}} \mathrm{X}$ macro system.

Copyright (c) 1993 by Pacific Journal of Mathematics 


\section{PACIFIC JOURNAL OF MATHEMATICS}

Volume $158 \quad$ No. $2 \quad$ April 1993

On the extension of Lipschitz functions from boundaries of subvarieties to

201 strongly pseudoconvex domains

K. ADACHI and HiRoshi KAJIMOTO

On a nonlinear equation related to the geometry of the diffeomorphism group 223

DaVid DaI-WAi BaO, JACQUES LAFONTAINE and TUdOR S. RATIU

Fixed points of boundary-preserving maps of surfaces

ROBERT F. BROWN and BRIAN SANDERSON

On orthomorphisms between von Neumann preduals and a problem of Araki 265

L. J. BUNCE and JOHN DAVID MAITLAND WRIGHT

Primitive subalgebras of complex Lie algebras. I. Primitive subalgebras of 273 the classical complex Lie algebras

\section{V. CHEKALOV}

$L^{n}$ solutions of the stationary and nonstationary Navier-Stokes equations in 293 $R^{n}$

\section{ZHI MiN CHEN}

Some applications of Bell's theorem to weakly pseudoconvex domains

XiAO JUN HUANG

On isotropic submanifolds and evolution of quasicaustics

STANISŁAW JANECZKO

Currents, metrics and Moishezon manifolds

SHANYU JI

Stationary surfaces in Minkowski spaces. I. A representation formula

JIANGFAN LI

The dual pair $(U(1), U(1))$ over a $p$-adic field

Courtney Hughes Moen

Any knot complement covers at most one knot complement 